\title{
Perception of Image Motion During Head Movement
}

\author{
LI LI \\ The University of Hong Kong \\ and \\ BERNARD D. ADELSTEIN and STEPHEN R. ELLIS \\ NASA Ames Research Center
}

\begin{abstract}
We examined human perception of head-referenced image motion during concurrent head movement. The visual stimulus was a checkerboard image in a head mounted display that moved from side-to-side. Observers rated the magnitude of the checkerboard motion while either rotating their head about a vertical axis (yaw), about a horizontal axis (pitch), or holding it still. In Experiment 1 , we tested four image oscillation frequencies $(0.25,0.5,1$, and $2 \mathrm{~Hz})$ while holding the head motion frequency constant at $0.5 \mathrm{~Hz}$. In Experiment 2, we tested three head motion frequencies $(0.25,0.5$, and $1 \mathrm{~Hz})$ while holding the image oscillation frequency constant at $1 \mathrm{~Hz}$. Across all image and head motion frequencies, perceptual sensitivity to image motion was reduced by about $45 \%$ during horizontal head movement. During vertical head movement, perceptual sensitivity was reduced by about $25 \%$ when head and image motion were of the same frequency. Compared with when the head was still, horizontal and vertical head movements produced a downward shift of about $10 \%$ in overall motion magnitude estimation response. Findings from this study provide virtual environment developers with a quantitative description of the influence of concurrent head movement on the perception of frontoparallel image motion.
\end{abstract}

Categories and Subject Descriptors: J.4 [Computer Applications]: Social and Behavioral Sciences—Psychology

General Terms: Human Factors, Design, Measurement, Performance

Additional Key Words and Phrases: Head movement, image motion, object motion, motion perception, VE latency

ACM Reference Format:

Li, L., Adelstein, B. D., and Ellis, S. R. 2009. Perception of image motion during head movement. ACM Trans. Appl. Percpt. 6, 1, Article 5 (February 2009), 15 pages. DOI = 10.1145/1462055.1462060 http://doi.acm.org/10.1145/1462055.1462060

\section{INTRODUCTION}

Studies of locomotion in both the real world and virtual environments (VEs) have reported that concurrent self-motion impairs visual perception of three-dimensional (3D) motion. For example, in the case of real-world driving, Probst et al. [1984] found that the time taken to detect an approaching or receding car increased by a factor of two to four when observers were in a moving as opposed to a stationary vehicle. For locomotion in a VE, Banton et al. [2005] asked observers to match the speed of an expanding

This research was supported by NASA's Space Human Factors Engineering Program and the University of Hong Kong Seed Funding Program for Basic Research.

Author's address: L. Li, Department of Psychology, The University of Hong Kong, Pokfulam Road, Hong Kong; e-mail: lili@hku.hk. B. D. Adelstein and S. R. Ellis, Human Systems Integration Division, NASA Ames Research Center, Moffett Field, CA 94035; e-mail: Bernard.D.Adelstein@nasa.gov; sellis@mail.arc.nasa.gov.

Permission to make digital or hard copies of part or all of this work for personal or classroom use is granted without fee provided that copies are not made or distributed for profit or commercial advantage and that copies show this notice on the first page or initial screen of a display along with the full citation. Copyrights for components of this work owned by others than ACM must be honored. Abstracting with credit is permitted. To copy otherwise, to republish, to post on servers, to redistribute to lists, or to use any component of this work in other works requires prior specific permission and/or a fee. Permissions may be requested from Publications Dept., ACM, Inc., 2 Penn Plaza, Suite 701, New York, NY 10121-0701 USA, fax +1 (212) 869-0481, or permissions@acm.org.

(c) 2009 ACM 1544-3558/2009/02-ART5 \$5.00 DOI 10.1145/1462055.1462060 http://doi.acm.org/10.1145/1462055.1462060 
flow pattern in a head-mounted display (HMD) to their walking speed on a treadmill, and found that observers often perceived their visually specified speed to be slower than their actual walking speed. Likewise, in studies asking observers to estimate the speed of an expanding flow pattern in an HMD, researchers found that subjective magnitude estimation of speed from optic flow could be reduced by active physical translation such as walking on a treadmill [Thurrell et al. 1998; Durgin et al. 2005; Pelah and Thurrell 2005].

Several theories have been proposed to explain the impairment of visual perception of 3D motion during concurrent physical translation. Among them, Barlow's [1990] "inhibition" theory states that highly correlated events such as walking and expanding optic flow mutually specify each other. The perceptual system uses this redundancy to modify its sensory coding in order to enhance the perception of novel events in the world (see also Pelah and Barlow [1996]). Neurophysiologically, these modifications in coding are produced by strengthening the inhibitory connections between simultaneously active neural units. According to Durgin et al. [2005], the mathematical operation underlying such coding changes is "subtractive" (i.e., a bias shift in response) rather than "divisive" (i.e., a gain or sensitivity reduction) in nature.

In the natural world, physical translation and head movement are highly linked during self-motion, such as we walk and turn our head to register objects by the side of the road. If physical translation inhibits visual perception of 3D motion, can head turns similarly affect visual perception of twodimensional (2D), frontoparallel motion? To answer this question, Wallach, Stanton, and Becker [1974] first studied how head turning influences perception of related environmental motion. They found observers perceived the environment as stationary if the added environmental motion was less than $3 \%$ of head turning motion regardless of whether the environmental motion was in the same or opposite direction to head movement. Wallach et al., attributed this perceived 3\% immobility range to a compensatory mechanism that strives to stabilize the world during self-induced motion. Later, Probst et al. [1986] reported increased manual reaction times for detecting the motion of a spot of light under different head rotations with respect to the body. Furthermore, Probst and Wist [1982] noted increased perceptual reaction time to head-referenced object motion when stimuli were presented to observers via an HMD. The observer's visual tracking of such head-referenced object motion during head rotation effectively suppressed the vestibulo-ocular reflex [VOR]. Normally, head movement activates the neck proprioceptors and the vestibular system in the inner ear, and the VOR induces compensatory eye movements to maintain the gaze direction on the object of interest in the environment as the head turns. The increased reaction time for motion detection observed in the absence of VOR signals suggests a perceptual inhibitory process based on inner ear or proprioceptive signals generated during head motion (e.g., Berthoz [1981]).

Despite the above findings, a number of issues remain unresolved. First, visual estimation of the magnitude of frontoparallel motion during head movement has not been fully measured. Second, the operational characteristic of the inhibitory mechanism for frontoparallel motion perception during head movement has not been clarified, that is, does head movement produce a bias shift in motion judgments as suggested by Durgin et al. [2005], or a reduction in perceptual sensitivity to motion, or a combination of the two? Although some of our previous work [Adelstein et al. 2006] has addressed these issues in part, a thorough analysis has yet to be conducted. Third, the extent to which concurrent head movement alters the perception of frontoparallel motion, and whether this alteration happens only when both are highly correlated, as proposed by Wallach [1987] and Barlow [1990], is unclear from the available data. The central aim of the current study is to clarify these issues.

In the current study, we used a magnitude estimation task similar to that of Durgin et al. [2005] to systematically assess the effect of head movement on frontoparallel image motion perception. Specifically, while wearing an HMD that showed a checkerboard image oscillating from side-to-side, observers 


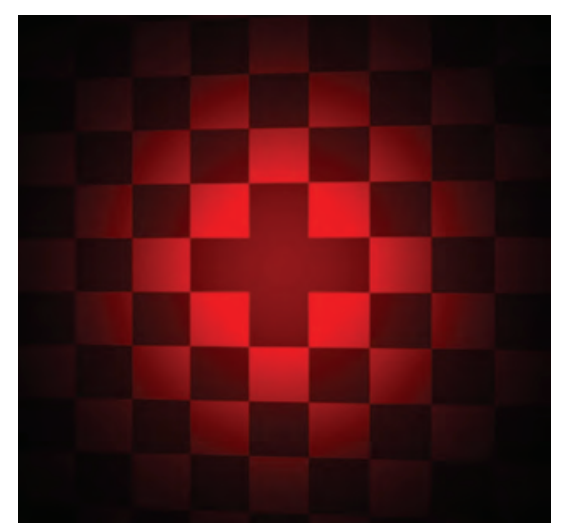

Fig. 1. The checkerboard pattern with a cross in the center.

were asked to judge the magnitude of the checkerboard motion while they yawed their head from side-to-side, pitched up and down, or held it stationary. The oscillatory image motion resembles image displacement errors seen by VE system users during back-and-forth head turns when excessive head tracking latency is present. We used oscillatory rather than constant direction linear image motion due to its relevance to help to further elucidate key aspects of latency perception in head-tracked VE systems. In Experiment 1, we varied sinusoidal image displacement amplitudes and tested four image oscillation frequencies to examine whether the effect of head movement on the perceived magnitude of image motion changed with image displacement amplitude and frequency. In Experiment 2, we used a complementary procedure and tested three head movement frequencies to examine whether head movement frequency affected the perceived magnitude of image motion. Given that side-to-side image motion is more similar to the relative motion caused by horizontal rather than vertical head movement, both the inhibition theory [Barlow 1990] and an accurate compensation process leading to perceived environmental stability [Wallach 1987] predict that horizontal head movement would have a larger suppression effect on image motion perception than would vertical head movement.

\section{GENERAL METHODS}

\subsection{Stimulus Generation and Control}

A red checkerboard pattern on a black background (Figure 1) was presented binocularly to participants via a Kaiser ProView 50ST HMD (Figure 2). The HMD provided VGA resolution $(640 \times 480$ pixels $)$ images with a $50^{\circ} \mathrm{H} \times 40^{\circ} \mathrm{V}$ field of view at a $60 \mathrm{~Hz}$ refresh rate. The bright red squares were set to RGB 8-bit (i.e., 255 max) triples of $(100,0,0)$, and the dark red squares were (50, 0, 0). The center of the checkerboard pattern included a cross that was composed of five dark red squares. Each checkerboard square subtended a visual angle of $6.8^{\circ}$ on a side. The Michelson luminance contrast between the two square colors was $58 \%$. A Gaussian mask $\left(\sigma=12^{\circ}\right)$ was used to darken the checkerboard toward the outer boundaries of the LCD image element in the HMD to obscure differential motion cues relative the HMD's LCD bezel.

During each trial, the checkerboard pattern was oscillated sinusoidally from side-to-side at a predetermined frequency with one of seven amplitudes of $0^{\circ}, 0.94^{\circ}, 1.88^{\circ}, 2.82^{\circ}, 3.76^{\circ}, 4.70^{\circ}$, and $5.64^{\circ}$ (one-half of peak-to-peak). The displacement amplitudes were selected to be nominally equivalent to the image slip (i.e., the lateral HMD-relative image motion caused by system time delay) experienced by participants in previous VE latency studies at NASA Ames [Adelstein et al. 2003, 2005]. The amplitudes are the image slip that would be seen during back-and-forth head turns for seven equally spaced 


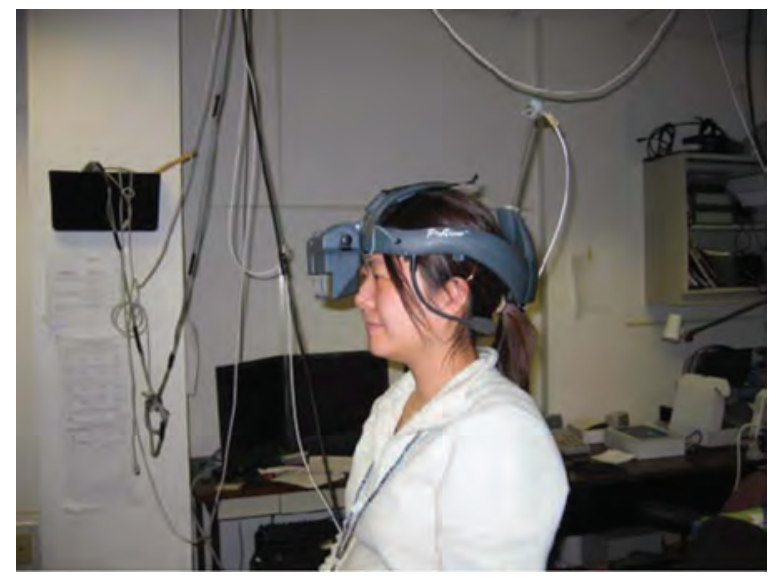

Fig. 2. Experimental set-up.

latency increments spanning zero to $120 \mathrm{~ms}$. Participants were instructed to fixate the central cross in the checkerboard image and, depending on the experiment block, either to keep their head stationary, yaw their head from side-to-side (horizontal head movement), or pitch up and down (vertical head movement) while making subjective magnitude estimates of the amplitude of the checkerboard motion. Uniform horizontal and vertical head movement frequency was paced by the beep of a computer-generated metronome that informed participants when to reverse head direction. Head movement began either from the left (horizontal) or at the bottom (vertical). An audible alarm based on Polhemus FasTrak readings of head angle reminded participants to limit yaw to approximately $\pm 15^{\circ}$ with respect to the straight-ahead direction, and pitch to $10^{\circ}$ above and $20^{\circ}$ below the horizontal. Figure 3 shows representative sample yaw and pitch head rotation data at three prespecified horizontal (yaw) and vertical (pitch) head movement frequencies $(0.25,0.5$, and $1 \mathrm{~Hz})$. As in Probst and Wist [1982], the image motion was relative to the HMD (i.e., head-referenced) rather than referenced to the external world. Thus, VOR-triggered compensatory eye movements were not involved in stabilizing the observer's gaze on the central cross in the checkerboard image. The HMD was covered with a black opaque hood and the laboratory room darkened to remove external visual stimuli.

\subsection{Procedure}

For trials in which the head was still, the checkerboard image was presented for $5 \mathrm{~s}$. For trials in which the head moved, the checkerboard image was presented beginning $1 \mathrm{~s}$ after the start of the head motion and then remained visible until the end of the trial's $6 \mathrm{~s}$ head movement interval. The HMD was otherwise "black," that is, at its minimum luminance. The phase of the image oscillation was randomized with respect to the head movement cycle for each trial in which the head moved.

Participants were instructed to rate the magnitude of the checkerboard motion by verbally announcing their evaluation as a percentage relative to a scale on which they were trained. The experiment monitor, who was blind to the specific experimental condition, then recorded their response and advanced to the next trial.

In order to anchor their individual responses, at the start of each experimental condition, participants were shown $0^{\circ}, 2.82^{\circ}$, and $5.64^{\circ}$ image motion amplitudes while their head was still. They were instructed to observe these motions and use them as references for 0,50 , and $100 \%$ of the maximum image motion magnitude. They could view these anchor stimuli as many times as they wished prior to 
Horizontal at $0.25 \mathrm{~Hz}$

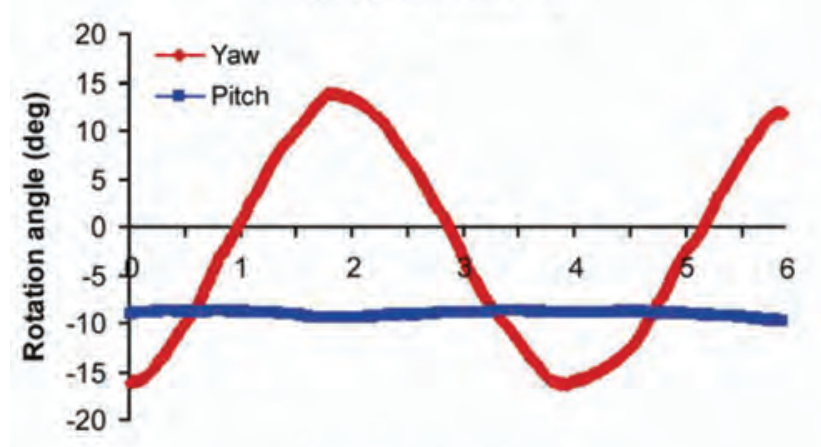

Horizontal at $0.5 \mathrm{~Hz}$

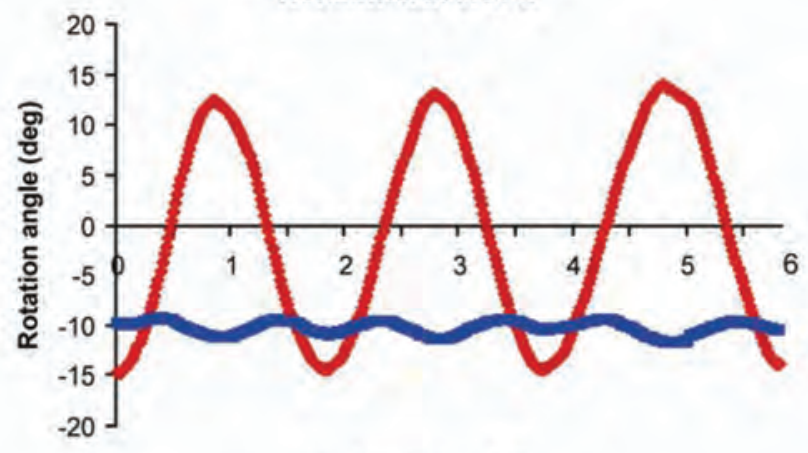

Horizontal at $1 \mathrm{~Hz}$

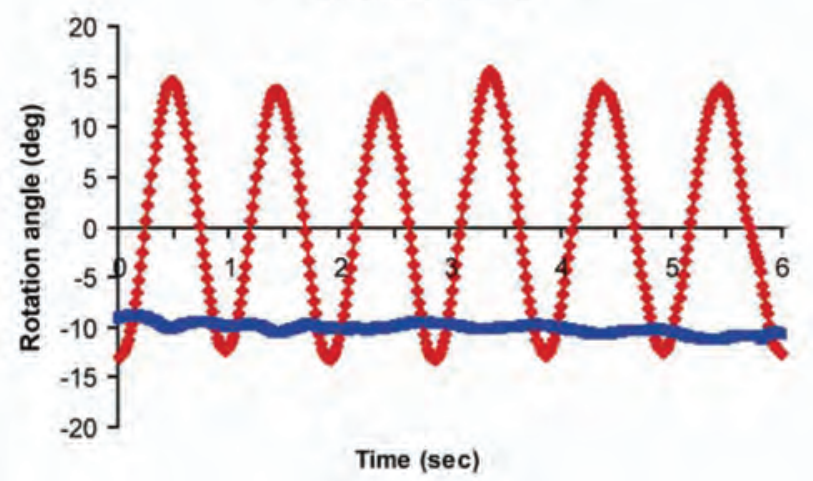

Vertical at $0.25 \mathrm{~Hz}$

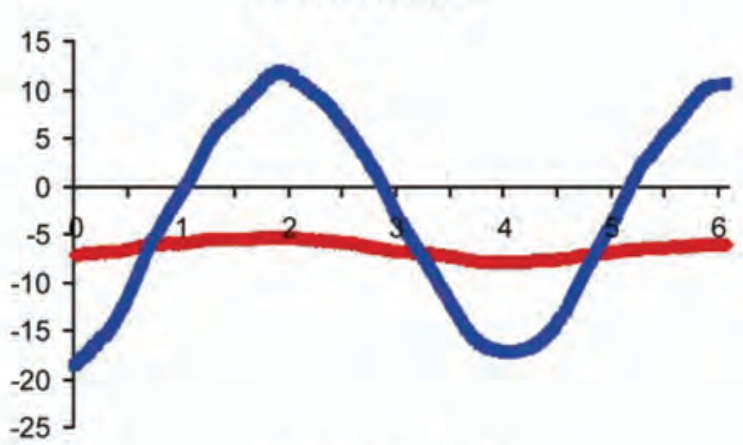

Vertical at $0.5 \mathrm{~Hz}$

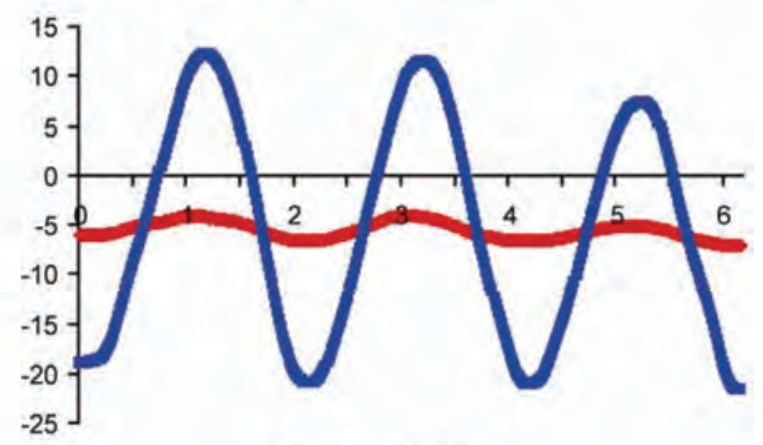

Vertical at $1 \mathrm{~Hz}$

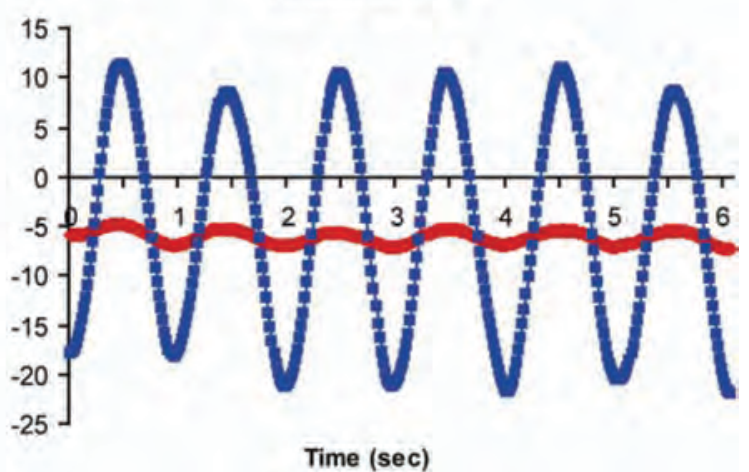

Fig. 3. Representative sample participant yaw and pitch head rotation data at $0.25,0.5$, and $1 \mathrm{~Hz}$ horizontal and vertical head movement frequencies. Note that the smaller amplitude oscillation in the minor axis is due, at least in part, to the orientation of motion sensor placement with respect to the head. The motion sensor signal itself was not used to drive the image motion in the experiment.

testing. Participants were also told that the image motion to be presented during testing might exceed $100 \%$ of the maximum image motion, so that responses greater than $100 \%$ were acceptable. Given that the image motion was relative to the HMD, we expected that participants would make their judgments with respect to the moving head (i.e., HMD) reference frame, not the fixed external world frame. The $0^{\circ}$ 


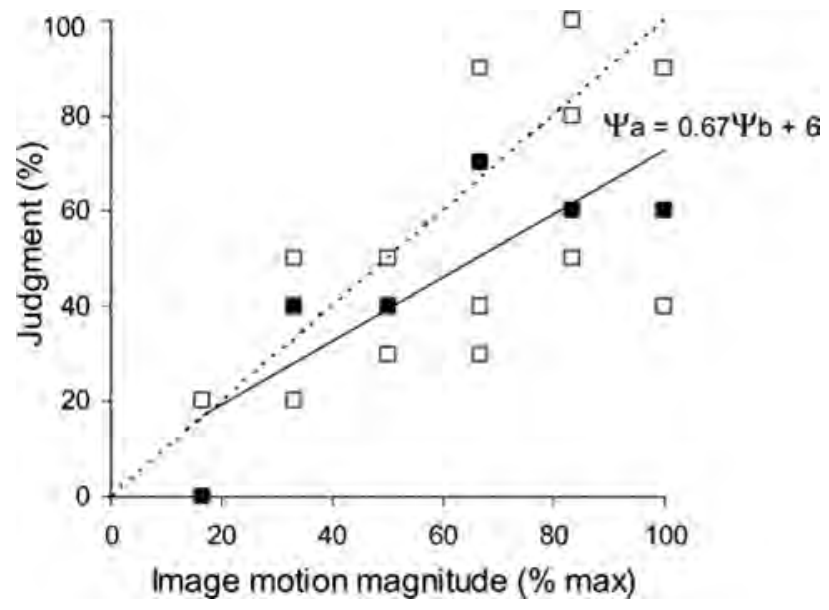

Fig. 4. Summary regression line for one participant's sample horizontal head movement condition. White squares represent 30 image motion judgments (some overlap) to the six non-zero input image motion amplitudes for $1 \mathrm{~Hz}$ image oscillation and $0.5 \mathrm{~Hz}$ head movement. Black squares are the medians of the five judgments at each stimulus level. The dashed line indicates perfect judgment.

image motion, that is, the stationary condition, repeatedly inserted throughout each testing condition, permitted us to verify whether participants maintained this reference frame.

\subsection{Data Analysis}

To investigate the impact of head motion on the subjective judgment of head-referenced image motion, we performed a linear regression analysis of judged image motion $\left(\Psi_{a}\right)$ against actual image motion $\left(\Psi_{b}\right)$ specified as follows:

$$
\Psi_{a}=K \Psi_{b}+A .
$$

The slope $K$ (in dimensionless units of \% divided by \%) represents the proportional increase of perceived motion magnitude for a given input stimulus, and the intercept $A$ (in units of $\%$ of maximum image motion at $5.64^{\circ}$ ) represents the estimation bias. A decrease in $K$ indicates a reduction in sensitivity, or gain, to physical changes in motion amplitude. A positive 'A' represents an overall over-estimation of the image motion, while a negative "A" represents an overall underestimation.

Before the regression analyses, we first converted input image motion amplitudes to percentages with $5.64^{\circ}$ image motion corresponding to $100 \%$. We then computed the median of the five responses provided by each participant at each image motion amplitude during a particular head movement condition for use in the regression analyses. Zero image motion amplitude was analyzed separately. Finally, we performed linear regressions of judgment response percentage, described by Equation 1, as a function of the six nonzero image motion amplitudes. With the rescaling of input motion to percentages, a regression with unity slope and zero intercept would correspond to perfect estimation. Figure 4 shows a regression line for one head movement condition from one participant. Subsequent analyses and discussion focus on the slopes and intercepts computed from the regressions.

\section{EXPERIMENT 1: IMAGE OSCILLATION FREQUENCY}

In this experiment, we varied the checkerboard image oscillation frequency while holding the head movement frequency constant at $0.5 \mathrm{~Hz}$. The purpose was to systematically examine the effect of head movement on image motion magnitude estimation at different image oscillation frequencies. Four 
image oscillation frequencies $(0.25,0.5,1$, and $2 \mathrm{~Hz})$ were tested, spanning the range of sinusoidal motion frequencies where visual tracking was possible without degraded visual acuity [Drazin 1962; Huddleston 1970].

\subsection{Participants}

Six observers (all naïve as to the specific goals of the study), aged between 19 and 36 years, participated in the experiment. All observers had normal or corrected-to-normal vision.

\subsection{Procedure}

Each observer participated in four experimental sessions over a two- or four-day period, with each session corresponding to one of the four image oscillation frequencies $(0.25,0.5,1$, and $2 \mathrm{~Hz})$. The testing order of image oscillation frequency was counterbalanced between observers. Each session consisted of four head movement condition blocks (initial head still, horizontal, vertical head movement, and final head still), with five repetitions for each of the seven image motion amplitudes in each block. Image motion amplitude presentation was randomized, with the same sequence followed for each block.

During each session, the head-still condition was tested first to get a baseline judgment scale. Participants then completed the horizontal and vertical head movement blocks in a counterbalanced order. A final, fourth head-still condition was run to determine whether there had been any shift in participant judgment criteria. Each head movement block typically lasted 8 min, with each experimental session completed in less than $40 \mathrm{~min}$. There was an interval of at least one hour between the four experimental sessions.

\subsection{Results}

Linear regressions of judgment percentages against the six non-zero image motion amplitudes were performed for each participant for each head movement condition at each image oscillation frequency. 94 of 96 regressions ( 4 image oscillation frequencies $\times 4$ head movement conditions $\times 6$ participants) were statistically significant $(r>0.73, p<0.05$ ); the other two were not and were therefore excluded from the subsequent data analyses. Of the 48 medians for zero image motion when the head was moving, all but one had $0 \%$ amplitude. The remaining one median was $5 \%$. This observation for the zero image motion amplitude during head movement indicates that participants were maintaining an HMD (i.e., head) reference frame when making all their judgments. Had they adopted a world reference frame, they would have reported motion.

3.3.1 Slope $(K)$. The mean slope averaged across six participants is plotted against image oscillation frequency in Figure 5(a). A paired $t$-test did not find the average slope for the "before" head-still condition $(1.05 \pm 0.07$, mean $\pm \mathrm{SE}$ across participants) significantly different from that for the "after" condition (1.02 \pm 0.07$)$, with $t_{23}=1.42, n s$. Therefore, we averaged the "before" and "after" slopes for each participant, yielding a single, combined "head-still" value. For all image oscillation frequencies, the mean of the combined head-still slope was not significantly different from the ideal unity slope to which participants were trained, indicating that participants' average perceptual sensitivity to image motion was essentially veridical when the head was stationary.

To determine the effects of head movement and image oscillation frequency on the regression slopes, we conducted a 3 (head movement condition) $\times 4$ (image oscillation frequency) repeated-measures ANOVA. Only the main effect of head movement was significant $\left(F_{2,10}=13.32, p<0.01\right)$. NewmanKeuls tests of the slopes for the three head movement conditions plotted in Figure 4(a) showed that the average slope for the horizontal head movement condition (0.57) was significantly lower than that for both the vertical head movement (0.87) and the head-still (1.04) conditions ( $p<0.01$, both cases). The 


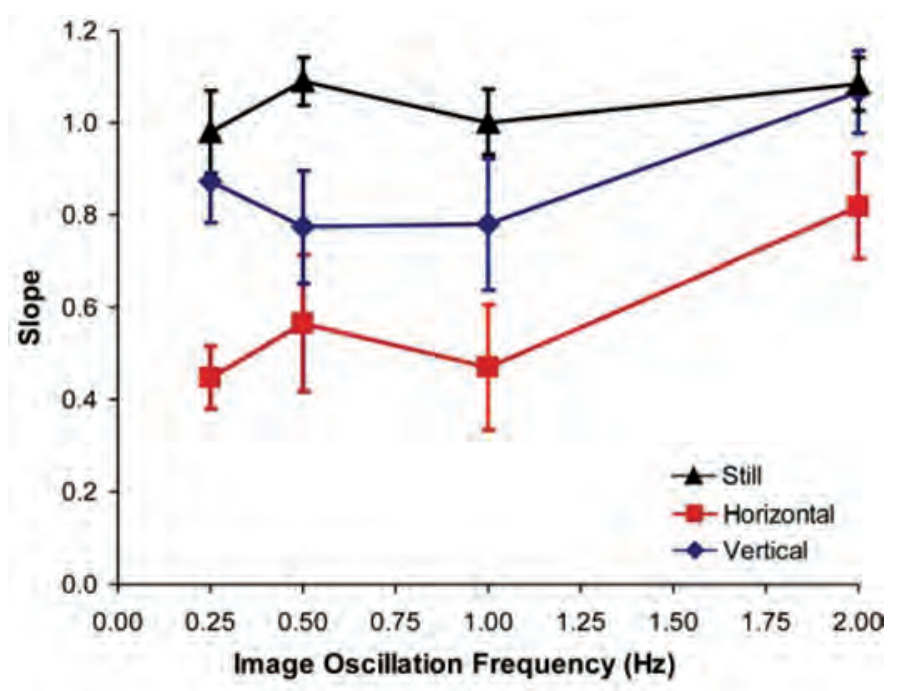

(a)

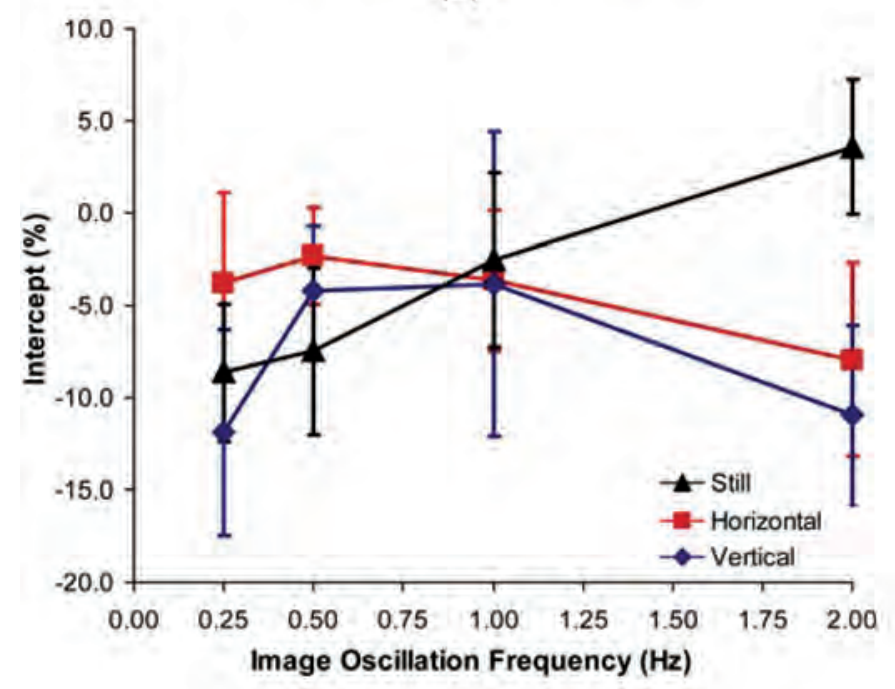

(b)

Fig. 5. (a) Mean slopes for the averaged before and after head-still, horizontal, and vertical head movement conditions against image oscillation frequency; (b) Mean intercepts for the averaged before and after head-still, horizontal, and vertical head movement conditions against image oscillation frequency. Error bars are SEs across six participants.

average slope for the vertical head movement condition was not significantly lower than when the head was still. Further Newman-Keuls tests revealed that while the slope of the horizontal head movement condition was significantly lower than that of the head-still condition at all four image oscillation frequencies (gain reduction range: 0.26 to $0.53, p<0.05$ ), the slope of the vertical head movement condition was only significantly lower than that of the head-still condition at the 0.5 and $1 \mathrm{~Hz}$ image oscillation frequencies (gain reduction: 0.26 and $0.22, p<0.05$ ). This indicates that the suppression effect of horizontal head movement on perceptual sensitivity to image motion did not depend on the 
image oscillation frequency, while the suppression effect of vertical head movement might be tuned with respect to the image motion oscillation frequency.

3.3.2 Intercept (A). The mean intercept averaged across six participants is plotted against image oscillation frequency in Figure 5(b). Again, a paired $t$-test did not find the average intercept for the "before" head-sill condition $(-5.18 \% \pm 4.22 \%$, mean $\pm \mathrm{SE}$ across participants) significantly different from that for the "after" condition $(-2.37 \% \pm 4.97 \%)$, with $t_{23}=-1.96, n s$, we hence averaged the "before" and "after" intercepts for each participant, yielding a single, combined "head-still" value. Although the mean of the combined head-still intercepts appeared to increase with image oscillation frequency, none was significantly different from the ideal zero-intercept (i.e., bias-free) response to which participants were trained.

A 3 (head movement condition) $\times 4$ (image oscillation frequency) repeated-measures ANOVA on the intercepts revealed that only the interaction between head movement condition and image oscillation frequency was significant $\left(F_{6,36}=5.17, p<0.001\right)$. There was a trend for the intercept to increase with image oscillation frequency in the head-still condition but not in either of the two head movement conditions. Newman-Keuls tests showed that only at the $2 \mathrm{~Hz}$ image oscillation frequency, the mean intercept for the head-still condition (3.58\%) was significantly higher than those for the horizontal $(-7.94 \%)$ and vertical $(-10.94 \%)$ head movement conditions $(p<0.01$ and $p<0.001$, respectively). The horizontal and vertical conditions were not significantly different from each other. This indicates that participants' image motion estimation bias was influenced by head movement only at the $2 \mathrm{~Hz}$ image oscillation, at which their overall motion magnitude estimation was respectively shifted $11.5 \%$ and $14.5 \%$ lower during separate horizontal and vertical head movement than during no head motion.

\section{EXPERIMENT 2: HEAD MOTION FREQUENCY}

The results from Experiment 1 indicate that horizontal head movement has a suppression effect on perceptual sensitivity to image motion across all four image oscillation frequencies tested, and vertical head movement suppresses perceptual sensitivity to image motion at the two middle image oscillation frequencies $(0.5$ and $1.0 \mathrm{~Hz})$. We suspect that the suppression effect with vertical head movement is tuned with the image oscillation frequency. On the other hand, the response bias in image motion estimation appears to be affected by head movement only at $2 \mathrm{~Hz}$, the highest image oscillation frequency tested. The difference in response bias observed between the head movement and the head-still conditions at $2 \mathrm{~Hz}$ appears to be attributable, at least in part, to the upward shift in the intercept for the head-still condition.

The current experiment therefore had three purposes. First, we further investigated the suppression effect of head movement on image motion perception using a complementary procedure, i.e., we varied the head movement frequency while holding the image oscillation frequency constant. Second, we further examined the frequency tuning of the suppression effect with vertical head movement. Lastly, we tested whether the response bias change observed in Experiment 1 was due to an intercept upward shift for the head-still condition. This time, we kept image oscillation frequency constant at $1 \mathrm{~Hz}$ and tested three head movement frequencies $(0.25,0.5$, and $1 \mathrm{~Hz})$. At faster head movement frequencies, participants would have difficulties to maintain the desired $30^{\circ}$ sinusoidal yaw or pitch head motion range. At slower head movement frequencies, participants' judgments based on only 5 s image visibility was impractical.

\subsection{Participants}

Eight observers (all naïve as to the specific goals of the study), aged between 20 and 45 years, participated in the experiment. All observers had normal or corrected-to-normal vision. However, two 
observers displayed large inconsistencies in their magnitude estimation for the "before" and "after" head-still conditions. One participant's overall motion magnitude estimation was about $50 \%$ greater in an "after" than in a paired "before" head-still condition block, while the other was about 75\% greater in a "before" than in a paired "after" head-still condition. Therefore, their data were excluded from the data analysis.

\subsection{Procedure}

Each observer participated in three experimental sessions over two or three days, with each session corresponding to one of the three head movement frequencies $(0.25,0.5$, and $1 \mathrm{~Hz})$. The image oscillation frequency was held constant at $1 \mathrm{~Hz}$. The testing order of head movement frequency was counterbalanced between observers. As in Experiment 1, each session consisted of four head movement condition blocks (initial head still, horizontal, vertical head movement, and final head still), with 35 trials in each block. The testing order of the horizontal and vertical head movement blocks in a session was also counterbalanced. Again, there was an interval of at least 1 hour between the three experimental sessions.

\subsection{Results}

Linear regressions of judgment percentages as a function of the six nonzero image motion amplitudes were performed for each participant for each head movement condition at each head movement frequency. All 72 regression ( 3 head movement frequencies $\times 4$ head movement conditions $\times 6$ participants) were statistically significant $(r>0.72, p<0.05)$. Of the 36 medians at zero image motion amplitude when the head was moving, all but 1 were reported as $0 \%$ amplitude. The remaining one median was $5 \%$. Again, this observation for the zero image motion amplitude during head movement indicates that participants were maintaining an HMD (i.e., head) reference frame when making all their judgments.

4.3.1 Slope $(K)$. The mean slope averaged across six participants is plotted against head movement frequency in Figure 6(a). A paired $t$-test did not find the average slope for the "before" head-still condition $(1.08 \pm 0.04$, mean $\pm \mathrm{SE}$ across participants) significantly different from that for the "after" condition $(1.09 \pm 0.05)$, with $t_{17}=-0.28, n s$. Hence, as in Experiment 1, we averaged the "before" and "after" slopes for each participant, yielding a single, combined "head-still" value. At the two lower head movement frequencies, the mean of the combined head-still slopes was not significantly different from the ideal unity slope response to which participants were trained. However, at the highest head movement frequency $(1 \mathrm{~Hz})$, the mean (1.085) of the combined head-still slopes was significantly different from the ideal unity slope $\left(t_{5}=3.85, p<0.05\right)$, indicating a slight increase in estimation gain or sensitivity (less than $9 \%$ ) for $1 \mathrm{~Hz}$ frequency head motion.

To determine the effects of both head movement condition and head movement frequency, we conducted a 3 (head movement condition) $\times 3$ (head movement frequency) repeated-measures ANOVA on the regression slopes. Only the main effect of head movement condition was significant $\left(F_{2,10}=\right.$ $35.09, p<0.0001$ ). Newman-Keuls tests of slopes for the three head movement conditions plotted in Figure 5(a) showed that the average slope for the horizontal head movement condition (0.65) was significantly lower than for the vertical head movement (0.96) and the head-still (1.09) conditions $(p<0.001$, both cases), and the average slope for the vertical head movement condition was significantly lower than for the head-still condition $(p<0.05)$. Further Newman-Keuls tests revealed that while the slope of the horizontal head movement condition was significantly lower than that of the head-still condition at all three head movement frequencies (gain reduction range: 0.26 to $0.57, p<0.05$ ), the slope of the vertical head movement condition was significantly lower than that of the head-still condition only when the head movement frequency was equivalent to the image oscillation frequency at $1 \mathrm{~Hz}$ (gain reduction: $0.22, p<0.05$ ). 


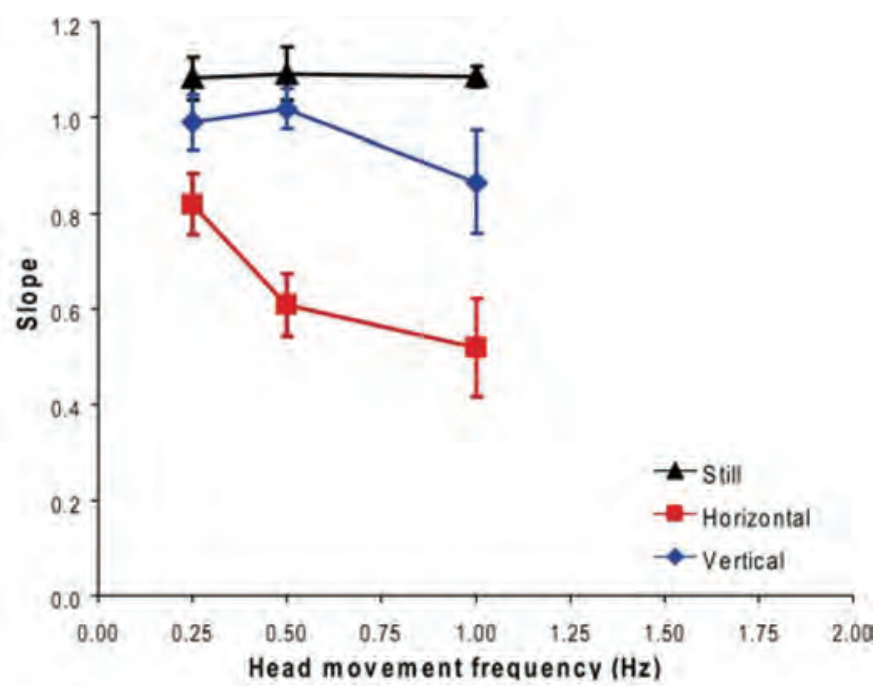

(a)

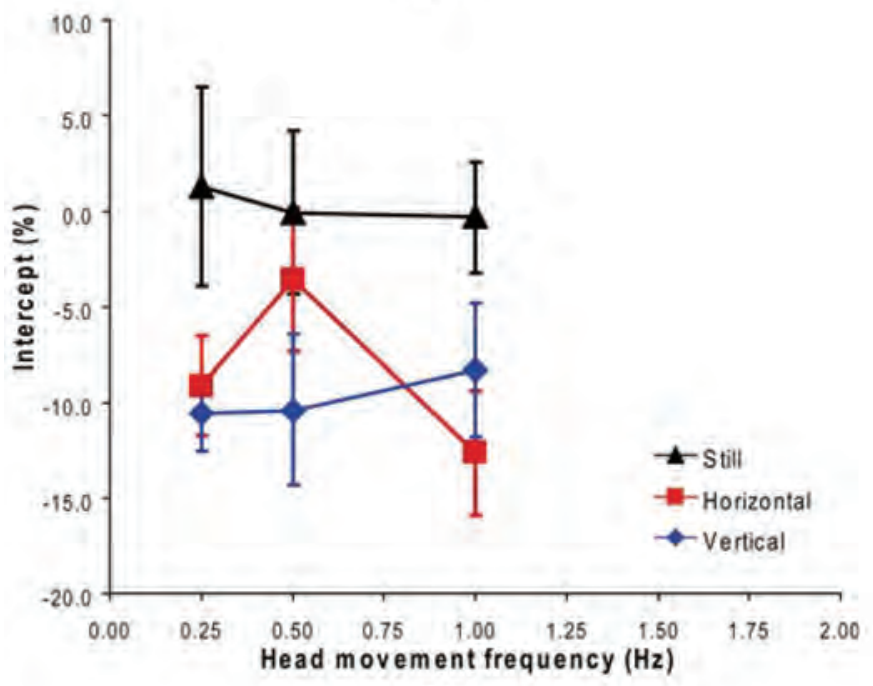

(b)

Fig. 6. (a) Mean slopes for the averaged before and after head-still, horizontal, and vertical head movement conditions against head movement frequency; (b) Mean intercepts for the averaged before and after head-still, horizontal, and vertical head movement conditions against head movement frequency. Error bars are SEs across six participants.

Looking at the results for horizontal head movement from Experiments 1 and 2, the suppression of perceptual sensitivity to horizontal image motion was observed for all the image and head oscillation frequency combinations studied. This sensitivity suppression, however, was not apparent for some headimage frequency combinations during vertical head movement, nor was it the same in both experiments. In particular, the significant gain reduction (0.22) with respect to the head-still condition for $0.5 \mathrm{~Hz}$ vertical head movement and $1 \mathrm{~Hz}$ image motion in Experiment 1 was not repeated for the matched condition in Experiment 2. In Experiment 2, the slope for $0.5 \mathrm{~Hz}$ vertical head movement (1.02) was not 
significantly different ( $p=0.46$ ) from when the head was still (1.09). Nevertheless, in both experiments, the sensitivity suppression during vertical head movement was consistently observed when the image and head motion were of the same frequency at 0.5 or $1 \mathrm{~Hz}$.

4.3.2 Intercept (A). The mean intercept averaged across six participants is plotted against head movement frequency in Figure 6(b). A paired $t$-test did not find the average intercept for the "before" head-still condition $(-0.55 \% \pm 4.87 \%$, mean $\pm \mathrm{SE}$ across participants) significantly different from that for the "after" condition $\left(1.19 \% \pm 4.51 \%\right.$, with $t_{17}=-0.61$, $n s$. We therefore averaged the "before" and "after" intercepts for each participant, yielding a single, combined "head-still" value. At all head movement frequencies, the mean of the combined head-still intercepts was not significantly different from the ideal zero-intercept (i.e., bias-free) response to which participants were trained.

A 3 (head movement condition) $\times 3$ (head movement frequency) repeated-measures ANOVA on the intercepts revealed that only the main effect of head movement condition was significant $\left(F_{2,10}=6.37\right.$, $p<0.05)$. Newman-Keuls tests showed that the mean intercept for the head-still condition $(0.32 \%)$ was significantly higher than those for the horizontal $(-8.43 \%)$ and vertical $(-9.74 \%)$ head movement conditions ( $p<0.05$, both cases). The horizontal and vertical conditions were not significantly different from each other. This indicates that across the three head movement frequencies tested, participants' image motion estimation bias was influenced by head movement. Their overall motion magnitude estimation was respectively shifted $8.8 \%$ and $10.1 \%$ lower during separate horizontal and vertical head movement than during no head motion.

\section{DISCUSSION}

Taken as a whole, the results from the two experiments quantitatively demonstrate the effects of head movement on the perceived magnitude of oscillatory frontoparallel image motion across a range of image and head motion frequencies. For $30^{\circ}$ peak-to-peak head movement at 0.25 to $1 \mathrm{~Hz}$, horizontal (yaw) head movement reduces perceptual sensitivity to image motion magnitude by about $45 \%$ from the veridical levels reported when the head is still. When the head motion frequency is equivalent to the image oscillation frequency at 0.5 or $1 \mathrm{~Hz}$, vertical (pitch) head movement reduces perceptual sensitivity by about $25 \%$ from the veridical levels. Furthermore, during both horizontal and vertical head movements, participants' overall motion magnitude estimation is shifted downward by approximately $10 \%$ of the maximum image motion amplitude, equivalent to about $0.6^{\circ}$. Our findings reinforce the observation that head movement has a suppression effect on frontoparallel motion perception and provide a basis for a quantitative model of image motion suppression during concurrent head movement.

\subsection{Gain vs. Bias Change in Inhibitory Processes}

Wallach [1987] proposed that the inhibitory effect of concurrent self-motion on visual perception of motion in the world reflects a compensation process that stabilizes the world during motor activities. For instance, head movement produces viewer-relative motions similar to those produced when the world is moving, but nonetheless we normally perceive the world as being stationary during head motion. The activation of this compensation process during head movement can be partially attributed to VORtriggered compensatory pursuit eye movements. As explained by Helmholtz [1962], the compensatory pursuit eye movements generate extra-retinal signals such as an efference copy to the brain so that the brain knows that the retinal image motion is due to the eye movement rather than the external object motion in the world. In our study, image motion was head-referenced. With the experimentally randomized phase relationship between the head and image motion cycles, if we presume that the eyes are nominally fixed in the head reference frame, image motion on the retina on average should be determined by the image motion in the HMD. This technique has neurophysiologically been shown 
effective in suppressing VOR-triggered compensatory eye movements in squirrel monkeys [Cullen et al. 1991]. The suppression effect of head movement observed in this study therefore lends support to the idea of a sensory inhibitory process using the vestibular or proprioceptive signals rather than the occulomotor signals (such as the efference copy from VOR-trigged compensatory eye movements) generated during head turns (e.g., Berthoz [1981]).

As a mechanism to implement Wallach's compensation process for self-motion, the inhibition theory of sensory correlation [Barlow 1990] has successfully explained the motion after-effect from treadmill walking [Pelah \& Barlow 1996]. According to this theory, highly correlated events mutually specify one another and consequently produce inhibitory interaction between their respective sensory coding for the enhancement of the perception of novel events. The perceived reduction of 3D motion speed during concurrent self-motion in this account "serves the functions of de-emphasizing predictable events in favor of detecting deviations from the norm" [Durgin et al. 2005]. Indeed, the extent of the perceived 3D speed reduction depends on the similarity between the concurrent physical activity and its expected visual consequences [Thurrell and Pelah 2005]. The findings from our current study support the inhibitory theory in general by showing that, in contrast to the frequency-specific suppression for vertical head movement, horizontal head movement, which is along the same axis as that of the side-to-side horizontal image motion, has a suppression effect on perceptual sensitivity to image motion across a broader range of head and image motion frequencies. Moreover, horizontal head movement more than doubles the reduction in perceptual sensitivity to image motion in comparison to the maximum seen for vertical head movement.

Barlow [1990] is unclear about whether the inhibitory process involves a perceptual bias shift in response or a gain (i.e., perceptual sensitivity) change as demonstrated, for example, by the inhibitory gain adjustment in contrast gain control [Heeger 1992]. Durgins et al. [2005] found that the inhibitory effect of physical translation on perceived speed of 3D motion could be modeled by a "subtractive" (i.e., a bias shift) operation. However, in the present study, we found that the suppression of frontoparallel image motion perception during head movement suggested both a sensitivity reduction and a response bias shift reflecting an overall underestimation of image motion across the three head movement frequencies tested. Aside from the experimental task and stimuli, the difference in our interpretation of the suppression mechanism may stem from the subjective magnitude estimation and data analysis procedures employed. Durgin et al. [2005] provided their participants with only one reference stimulus, termed "100," for comparison, while we anchored three points $(0,50$, and $100 \%)$ of our estimation scale to linearize participants' responses. Additionally, Durgin et al.'s logarithmic transformation of data, based on an assumption of an underlying Steven's power-law mechanism for perceptual suppression of image motion, makes it impossible to interpret simultaneous gain and bias effects as one otherwise would from concurrent changes in slope and intercept from linear regression analyses of untransformed data.

\subsection{Motion Suppression Effect and VE Latency}

VE latency, the delay between a user's input movement and the time when the movement's consequences are rendered in the display, induces dynamic errors in image position during and immediately following user input movement. These errors cause virtual objects to drift or "slip" from their expected real-world stable locations [Adelstein et al. 2003; Azuma and Bishop 1994]. When sufficiently large, the errors can hamper users' perception and performance in the VE. In prior studies, Adelstein et al. [2005] found that observers make use of the velocity rather than the displacement associated with such image slip to discriminate the presence of latency.

While we did not manipulate VE latency per se in the experiments reported here, the visual stimuli presented did introduce image motion characteristics very similar to those experienced for the 
range of latencies employed in our prior studies [Adelstein et al. 2003, 2005]. The suppression of headreferenced image motion perception during head movement demonstrated by our current study may explain why participants previously appeared more sensitive to image slip velocity rather than displacement [Adelstein et al. 2005]. When VE latency is present, maximum image slip velocity for sinusoidal head movement profiles occurs during direction reversals, as head velocity approaches zero. Conversely, maximum image slip displacement occurs at the center of travel (between the opposite reversal points) when head movement is fastest. Consequently, the peak image velocity intervals that participants associated most strongly with VE latency in our previous studies [Adelstein et al. 2003, 2005] corresponded to the periods of slowest instantaneous head movement, when the perception of image motion was least suppressed.

Latency mitigation schemes such as prediction [Azuma and Bishop 1994] potentially offer means to diminish the salience of latency artifacts, but are themselves imperfect. The schemes can introduce other motion errors such as overshoot and high-frequency noise that can have perceptual consequences as distracting as those of the original latency [Jung et al. 2001]. While the dynamic characteristics of errors introduced by VE latency and predictive compensation are easily described analytically, such imperfections remain a significant challenge in the implementation of successful VE systems [Brooks 1999].

The current observation that perception of HMD-relative, head-referenced image motion is partially suppressed regardless of whether the viewed image motion is at the same frequency or even in the same direction as the input head movement has two immediate practical implications for the management of latency in VE systems. First, designers of latency compensation schemes might consider defining filter parameters not in terms of raw head movement but instead with respect to image motion error as perceived by the user, i.e., with diminished sensitivity during head movement. Second, designers might simply choose to temporarily de-emphasize or even deactivate latency compensation while the head is moving.

\section{REFERENCES}

Adelstein, B. D., Burns, E. M., Ellis, S. R., AND Hill, M. I. $2005 . \quad$ Latency discrimination mechanisms in virtual Environments: velocity and displacement error factors. In Proceedings of the 49th Annual Meeting of the Human Factors and Ergonomics Society. HFES, Santa Monica, CA, 2221-2225.

Adelstein, B. D., Lee, T. G., And Ellis, S. R. 2003. Head Tracking Latency in Virtual Environments: Psychophysics and a Model. In Proceedings of the 47th Annual Meeting of the Human Factors and Ergonomics Society. HFES, Santa Monica, CA, 2083-2087.

Adelstein, B. D., Li, L., Jerald, J. J., And Ellis, S. R. 2006. Suppression of head-referenced image motion during head movement. In Proceedings of the 50th Annual meeting of Human Factors and Ergonomics Society. HFES, Santa Monica, CA.

Azuma, R. AND Bishop, G. 1994. Improving static and dynamic registration in an optical see-through display. In Proceedings of the SIGGRAPH. IEEE, Los Alamitos, CA, 197-204.

Banton, T., Stefanucci, J., Durgin, F., Fass, A., And Proffitt, D. $2005 . \quad$ The perception of walking speed in a virtual environment. Presence 14, 394-406.

BARLow, H. B. $\quad$ 1990. A theory about the functional role and synaptic mechanism of visual aftereffects. In C. Blakemore, Ed., Vision: Coding and Efficiency. Cambridge University Press, Cambridge, UK, 363-375.

Berthoz, A. 1981. Intersensory interaction in motion perception. In J. Long and A. Baddeley, Eds., Attention and Performance, Vol. IX. Lawrence Erlbaum Associates, Hillsdale, NJ, 27-45.

Brooks, F. P., JR. 1999. What's real about virtual reality? IEEE Comput. Graph. Appl. 19, $16-27$.

Cullen, K. E., Belton, T., And Mccrea, R. A. 1991. A non-visual mechanism for voluntary cancellation of the vestibulo-ocular reflex. Exper. Brain Resear. 83, 237-252.

DrazIN, D. H. 1962. Factors affecting vision during vibration. Research 15, 275-280.

Durgin, F. H., Gigone, K., And Scott, R. 2005. Perception of visual speed while moving. J. Exper. Psych.: Hum. Percep. Perform. $31,339-353$.

HeEger, D. J. 1992. Normalization of cell responses in cat striate cortex. Visual Neuroscience 9, $181-197$. 
von Helmholtz, H. 1962. Physiological Optics Vol III (treatise from $3^{\text {rd }}$ Germen edition of 1910). Dover, New York.

Huddleston, J. H. F. 1970 . Tracking performance on a visual display apparently vibrating at one to ten Hertz. J. Appl. Psych. $54,401-408$.

JUNG, J. Y., AdELSTEIN, B. D., AND ElLis, S. R. 2000. Discriminability of prediction artifacts in a time delayed virtual environment. In Proceedings of the 44th Annual Meeting of the Human Factors and Ergonomics Society. HFES, Santa Monica CA, 499-502.

Pelah, A. ANd Barlow, H. B. $\quad$ 1996. Visual illusion from running. Science 381, 283.

Pelah, A. And Thurrell, A. E. I. 2005. Matching visual and non-visual signals: Evidence for a mechanism to discount optic flow during locomotion. In Proceedings of the SPIE-IS\&T Electronic Imaging. Bellingham, WA, 5666, 434-448.

Probst, T., Brandt, T., and Degner, D. 1986. Object-motion detection affected by concurrent self-motion perception: psychophysics of a new phenomenon. Behav. Brain Resear. 22, 1-11.

Probst, T., KrafczyK, S., Brandt, T., AND Wist, E. R. 1984 . Interaction between perceived self-motion and object-motion impairs vehicle guidance. Science 225, 536-538.

Probst, T. ANd Wist, E. R. 1982 . Impairment of object motion perception during head movements. Perception 11 s, A33.

Thurrell, A. E. I., Pelah, A., And Distler, H. K. 1998. The influence of non-visual signals of walking on the perceived speed of optic flow. Perception 27s, 147.

Wallach, H. 1987. Perceiving a stable environment when one moves. Annual Rev. Psych. 38, 1-27.

Wallach, H., Stanton, L., and Becker, D. 1974. The compensation for movement-produced changes in object orientation. Percep. Psychophy. 15, 339-343.

Received December 2006; revised August 2007, February 2008; accepted March 2008 